\title{
Gender difference in drinking among the urban squatters of Nepal
}

\author{
Pushpa Thapa ${ }^{1^{*}}$, Shiva Raj Mishra ${ }^{2}$ \\ ${ }^{1}$ Nepal Health Research Council, Ramshah Path, Kathmandu, Nepal ${ }^{2}$ Nepal Development Society, Bharatpur-10, Chitwan, Nepal
}

\begin{abstract}
Background: Globally, more men drink than women. However, we remain unknown about this phenomenon among the urban poor of Nepal. The current study investigated gender differences in drinking among the urban poor residing in urban squatter settlements of Nepal.

Methodology: We approached 422 households of four squatter settlements of Kathmandu Valley, using modified Gender, Alcohol, and Culture: an International Study questionnaire. Following cross-sectional design, we executed study from November, 2013 to March, 2014. Bivariate and multivariable logistic regression was done in $\mathrm{R}$ version 3.1.2.
\end{abstract}

Results: Odds of being current drinker (adjusted odds ratio: 5.86, 95\% CI: 2.50-13.72) was higher in men than the women. Men were also more likely to be frequent drinkers (adjusted odds ratio: 6.61, 95\% CI: 1.45-

Received:

29 June 2015

Revised:

01 December 2015

\section{Accepted:}

12 December 2015

\section{${ }^{*}$ Correspondence:} pushpathapa242@gmail.com Nepal Health Research Council, Ramshah Path, GPO Box 7626, Kathmandu, Nepal 30.11) than their women counterparts. Men and women did not differ significantly in contexts of drinking and types of drinking. Men also carried higher possibility of being current drinker in various strata of socio-demographic characteristics. The respective crude odds ratio were accordingly: (18-24 years: 12.73, 95\% CI: 4.13-40.98); (25-44 years: 9.94, 95\% CI: 4.54-22.13); (45-65 years: 8.23, 95\% CI: 3.70-18.57); (dalit and disadvantaged janajati:9.80, 95\% CI: 5.61-17.21); ( upper caste: 9.00, 95\% CI: 3.25-25.78); ( Hindu: 8.67, 95\% CI: 4.79-15.80); (non-Hindu: 13.64, 95\% CI: 5.46-34.88); (secondary and below education: 10.22, 95\% CI: 6.01-17.46); (above secondary education:7.00, 95\% CI: 2.05-25.06); (employed: 3.94, 95\% CI: 1.74-8.99); (unemployed: 14.08, 95\% CI: 7.45-26.84); (married: 9.79, 95\% CI: 95\% CI: 5.27-18.33); (unmarried and others: 9.33, 95\% CI: 4.18-21.15).

Conclusion: The study revealed significant gender differences in drinking. Gender sensitive and specific alcohol interventions should be planned and implemented covering urban squatter settlements.

Keywords: Drinking, Gender difference, Kathmandu Valley, Squatter settlements, Urban poor

Suggested citation: Thapa P, Mishra SR. Gender difference in drinking among the urban squatters of Nepal. Health Prospect. 2016;15(1):11-15.

Tweetable abstract: Alcohol use is almost seven times more common among Men in Nepal's urban squatters than their counterparts.

\section{Introduction}

Drinking is a global public health problem claiming 3.3 million lives in 2012. Figure of burden of disease and injury attributable to drinking was $7.4 \%$ for men and $2.3 \%$ for women (1).

Worldwide, about two billion people consume alcoholic beverages, with marked gender differences (2). Significant gender gap on drinking patterns has been well documented around the world (3-5). Nevertheless, sizes of these gender differences vary from society to society.

Alcohol has been part of various cultures, since long time as means of differentiating, symbolizing, and regulating gender roles (6). Drinking has been traditionally viewed as a masculine behavior across societies of the world (7).

Practice of alcohol in Nepal has a long history. Drinking culture of Nepal comes under the ambivalent culture, with both sternly negative and prohibitive attitudes towards drinking (8).

STEPS (STEPwise approach to surveillance) Survey identified $17.40 \%$ current drinkers, with men outnumbering women by ratio of four to one (9). Oli et al., in their study in Sinamangal slum of Kathmandu Valley, came out with visible gender gap in drinking: $58.00 \%$ men and $24.90 \%$ women (10). Almost all studies among different segments of the population of Nepal showed consistent result, with limited share of women in drinking (11-14).

An increasing body of literature in developing countries showed consistent gender differences in drinking of the urban poor (10, 15-19). Gender difference related data on drinking of urban poor's is scarce in Nepal. Research on gender and alcohol is imperative to address costly biases in how societies attempt to control or reduce alcohol related problems, because of gender stereotypes regarding drinking (20). Gender roles encouraging heavy drinking as masculinity may encourage men to perceive drinking as normal (21), even though it may lead to social and public health consequences. Likewise, assumption that women 
hardly drink may result into the underestimation of women's drinking problem. Moreover, health consequences of women's drinking, especially during pregnancy are too hard to ignore with the outcomes like low birth weight, pre-term birth (22), fetal alcohol spectrum disorders (23), to birth defects (24). It's also not uncommon for alcoholic women to suffer violence (25), depression, and reproductive health problems (26), especially for heavy drinkers.

Examining gender differences in drinking is therefore crucial to draw gender sensitive and specific alcohol interventions. We determined an independent effect of gender on drinking among the urban poor of squatter settlements of Nepal.

\section{Methods}

Study design and setting

We carried out study from November 2013 to March 2014, following descriptive, cross-sectional design. Squatter settlements of Kathmandu Valley constituted the study site. Of these settlements, four (Shankamul, Ramhiti Improved, Manohara Bhaktapur, and Radhakrishna Chowk) were chosen randomly. Kathmandu, the capital city of Nepal is inhabited by 12,726 urban poor with a per capita income of below $\$ 1$ per day (27). The city is occupied by 40 squatter settlements and five slums (27).

\section{Sampling technique}

Sample size consisted of 422 urban poor aged 18 years and above, which came from formula $\mathrm{N}=\mathrm{Z}^{2} \mathrm{PQ} / \mathrm{d}^{2}$ (28) (50\% assumed for conservative sample size estimates, with $5 \%$ allowable error, $95 \%$ confidence level, and adding 10\% non-response rate). We applied multistage random sampling method to select samples. Firstly, four out of 40 squatter settlements were selected as primary sampling units. Secondary sampling units comprised households of selected squatter settlements. We sampled households purposively due to lack of sampling frame. From each selected household, we interviewed one person aged 18 years and above. In case of availability of more than one person meeting the inclusion criteria, we used the lottery method to select one by chance. The study excluded mentally challenged, severely ill, and those living in a house for less than six months.

\section{Data collection procedure}

The study assessed drinking pattern with modified GENACIS (Gender, Alcohol, and Culture: an International Study) questionnaire (29). Only the questions relevant to the study objectives were included from the GENACIS. We did backto-back translations of the questionnaire into Nepali and then English to ensure no serious distortion in the translation process. The questionnaire was fragmented into different sections: drinking pattern, frequency, contexts, types, and sociodemographic characteristics. Three female interviewers trained by principal investigator underwent face-to-face interview.

\section{Operational definition of variables}

Study examined drinking habit with reference period of last 12 months preceding the survey (2). Dependent variables were namely current drinking, frequent drinking, and heavy drinking. Independent variable of major interest was gender. List of covariates included age, education, ethnicity, religion, occupation, marital status, and family history of alcohol. We considered respondents as a current drinker, if they reported drinking in the last 12 months prior to survey (2). Lifetime abstainers consisted of those who never drank alcohol (2). Further, frequent drinkers and heavy drinkers were assessed among current drinkers. Study defined frequent drinkers as those who drank three or more times a week, irrespective of number of glasses. Those having five or more drinks in a single occasion were categorized as heavy drinkers (30). We categorized types of drinking as jaad/chyang, beer, homemade raksi, local raksi available at market, distillery products, and combination (more than one type of drink) (8). Definition of contexts of drinking covered an occasion, place, companion, and time of drinking $(31,32)$.

\section{Data analysis}

We used SPSS full version 19 to enter data. Data were then transported to $\mathrm{R}$ version 3.1.2 for required analysis. Bivariate analysis (Chi-square test and Fischer's exact test) examined an association between gender and patterns of drinking (current drinking, frequent drinking, and heavy drinking), types of drinking, and contexts of drinking accordingly.

Dependent variables with p-value $<0.05$ were subjected into multivariable analysis to identify an independent effect of gender on drinking, with effects of other potentially confounding factors simultaneously being controlled. In the final model, we adjusted the effect of age, education, ethnicity, religion, occupation, marital status, and family history of alcohol. The logistical regression model was used for multivariable analysis. Further, we tested gender difference of current drinking in different strata of socio-demographic characteristics. We presented results with odds ratio (OR) and 95\% confidence interval (CI).

\section{Research ethics}

An independent Ethical Review Board of Nepal Health Research Council provided ethical clearance. We explained research objectives, procedure, confidentiality, risks, and benefits to respondents, before obtaining verbal consent. Those providing verbal consent to participate in the study after knowing details of research were recruited into the study.

\section{Results}

\section{Socio-demographic characteristics}

Our sample comprised of $46.70 \%$ men and $53.30 \%$ women. The majority fell under group of $25-44$ years $(42.65 \%)$, disadvantaged janajati (75.36\%), Hindu (67.77\%), secondary and below education holder (83.66\%), unemployed (64.22\%), and married (63.03\%) (Table not shown).

\section{Differences in drinking patterns, types, and contexts by gender}

Bivariate analysis suggested significant differences in patterns of drinking by gender (Table 1). Types of drinking did not differ significantly with gender. Our data also lacked sufficient evidence to show an association between contexts of drinking and gender (Table not shown).

Not surprisingly, the study came out with an evident gender differences in drinking patterns. The probability of being current drinker $(p=0.000)$ and frequent drinker $(p=0.015)$ were 


\begin{tabular}{|lccc|}
\hline Table 1: Drinking patterns by gender & & & \\
\hline Pattern of drinking & Crude OR (95\% CI) & Adjusted OR(95\% CI) & Nagelkerke R square \\
\hline Current drinker & $5.47(2.45-12.33)$ & $5.86(2.50-13.72)^{*}$ & \\
Male & & 19.8 & 0.293 \\
Female & & $6.61(1.45-30.11) \#$ \\
Frequent drinker & $0.65(0.26-1.54)$ & & -159 \\
Male & & & - \\
Female & & & - \\
Heavy drinker & $1.67(0.63-4.36)$ & & \\
Male & & & \\
Female & & & \\
\hline
\end{tabular}

*significant at $<0.001$; \# significant at $<0.05$; adjusted for age, education, ethnicity, religion, occupation, marital status, and family history of alcohol

\begin{tabular}{|c|c|c|c|c|c|}
\hline \multirow[t]{3}{*}{ Demographics } & \multirow{2}{*}{\multicolumn{2}{|c|}{$\begin{array}{c}\text { Male } \\
\text { Curret drinker }\end{array}$}} & \multirow{2}{*}{\multicolumn{2}{|c|}{$\begin{array}{c}\text { Female } \\
\text { Current drinker }\end{array}$}} & \multirow[t]{3}{*}{ Crude OR ( $95 \% \mathrm{CI})$} \\
\hline & & & & & \\
\hline & Yes $(n=130)$ & No $(n=67)$ & Yes $(n=38)$ & No $(n=187)$ & \\
\hline \multicolumn{6}{|l|}{ Age in years } \\
\hline $18-24$ & 26 & 14 & 7 & 48 & $12.73(4.13-40.98)^{\star}$ \\
\hline $25-44$ & 52 & 33 & 13 & 82 & $9.94(4.54-22.13)^{*}$ \\
\hline $45-64$ & 52 & 20 & 18 & 57 & $8.23(3.70-18.57)^{*}$ \\
\hline \multicolumn{6}{|l|}{ Ethnicity } \\
\hline $\begin{array}{l}\text { Dalit and disadvantaged } \\
\text { janajati }\end{array}$ & 94 & 47 & 30 & 147 & $9.80(5.61-17.21)^{*}$ \\
\hline Upper caste & 36 & 20 & 8 & 40 & $9.00(3.25-25.78)^{*}$ \\
\hline \multicolumn{6}{|l|}{ Religion } \\
\hline Hindu & 92 & 54 & 23 & 117 & $8.67(4.79-15.80)^{*}$ \\
\hline $\begin{array}{l}\text { Non-hindu (Muslim, Bud- } \\
\text { dhist, Christian) }\end{array}$ & 38 & 13 & 15 & 70 & $13.64(5.46-34.88)^{*}$ \\
\hline \multicolumn{6}{|l|}{ Highest education } \\
\hline Upto secondary & 109 & 53 & 32 & 159 & $10.22(6.01-17.46)^{*}$ \\
\hline Above secondary & 21 & 14 & 6 & 28 & $7.00(2.05-25.06)^{*}$ \\
\hline \multicolumn{6}{|l|}{ Occupation } \\
\hline Employed & 24 & 29 & 17 & 81 & $3.94(1.74-8.99)^{*}$ \\
\hline Unemployed & 106 & 38 & 21 & 106 & $14.08(7.45-26.84)^{*}$ \\
\hline \multicolumn{6}{|l|}{ Marital status } \\
\hline Married & 85 & 45 & 22 & 114 & $9.79(5.27-18.33)^{*}$ \\
\hline Unmarried and others & 45 & 22 & 16 & 73 & $9.33(4.18-21.15)^{*}$ \\
\hline
\end{tabular}

Note: Reference category is female; ${ }^{*}$ significant at $\mathrm{p}<0.001$

significantly higher in men. Significant gender differences were observed among current drinkers $(\mathrm{aOR}=5.86,95 \% \mathrm{CI}: 2.50$ 13.72) and frequent drinkers $(\mathrm{aOR}=6.61,95 \% \mathrm{CI}: 1.45-30.11)$. We found the odds of heavy drinking 1.67 times higher in men, compared to women; however, it lacked statistical significance (95\% CI: 0.63-4.36) (Table 1).

Significant gender differences were visible within different age groups. 18-24 years men were 12.73 times (95\% CI: 4.13 40.98) more likely to be current drinkers than $18-24$ years women. We observed alike result in 25-44 years, with 9.94 times (95\% CI: 4.54-22.13) higher likelihood in men. Similar was the finding among 45-64 years age group with 8.23 times (95\%
CI: 3.70-18.57) higher likelihood in men than their women counterparts. Upper caste men were 9.00 times (95\% CI: 3.2525.78) more likely to be current drinkers than upper caste women. So, were dalit and disadvantaged janajati men with 9.80 times higher (95\% CI: 5.61-17.21) odds of being current drinkers than women of their caste. Hindu men were 8.67 times (95\% CI: 4.79-15.80) and non-Hindu men were 13.64 times (95\% CI: 5.46-34.88) more likely to be current drinkers than women of their religion. Men holding up to secondary education were 10.22 times (95\% CI: 6.01-17.46) more likely to be current drinkers than women of similar education level. Unemployed men were 14.08 times (95\% CI: 7.45-26.84) more likely to be 
current drinkers than unemployed women. Likewise, married men were 9.79 times (95\% CI: 5.27-18.33) more likely to be current drinkers than married women (Table 2).

\section{Discussion}

Our findings suggested higher drinking behavior of men, compared to women. Consistent results have been reported in number of studies, conducted among urban poor, across the globe (16-18). The finding also went in line with the Nepalese study featuring Sinamangal slum of Kathmandu Valley (10).

We also detected significant gender differences in various strata of socio-demographic characteristics like age group, education, occupation, ethnicity, religion, and marital status. However, we did not find interaction effect by age and gender on drinking habit. In Nepal, like in other developing nations, drinking is traditionally regarded as a male issue (33). In this light, possibility of underestimation of women's drinking habit could not be ruled out, which might have resulted in significant gender differences in drinking. Nevertheless; pertaining to wider CI in adjusted estimates, low possibility of good estimates of magnitude of the effect cannot be ignored.

As for heavy drinking, types of drinking, and contexts of drinking, men and women in our study did not differ significantly. Very few samples of women in each category of types and contexts may not be enough to detect an association.

Studying gender differences in drinking is essential to address the negative effects created by gender role on treatment and prevention of alcohol-related problems (34). As drinking is regarded manly behavior (33), women are more likely to underreport drinking, thus drawing less attention of policy makers and program planners. Equally, supposition of negligible drinking among women, may enhance the chances of their health problems being under looked (35). This could have profound impact on maternal and neonatal health.

Masculinity being related to drinking is expected to encourage male drinkers to perceive drinking as normal behavior and deny problems due to drinking (21). As a consequence, drinkers may have to face violence $(36,37)$ and non communicable diseases (38). What matters most is, the vulnerability of women to interpersonal violence (37) and risky sexual behaviors (39), because of drinking behavior of their partners. Owing to drinking environment in family, children are also forced to suffer various social problems like family violence (40) and child abuse (8).

This is by far, the first systematic study of gender differences of drinking among the urban poor of Nepal. The findings carry implication for gender sensitive alcohol programs, as they indicated higher odds of drinking among men. This may have negative impact on family and community with the high cost of drinking (41), brought by social consequences of drinking (42). On top of that, concerns are widespread for health and social consequences of urban poor, because of less access to resources (43). Matter of equity therefore arises, to address gender differences of drinking among the urban squatter settlements of Nepal.

\section{Conclusion}

Drinking among the urban poor, markedly differed by gender. The findings underscore the necessity of gender sensitive and specific alcohol prevention and treatment programs in the squatter settlements of Nepal. The question of what drive gender differences in drinking among this population still remains largely unexplained. Studies with larger and representative samples should be on the floor to answer this important unanswered question.

\section{Limitations}

We must admit that our study had its share of limitations. The study suffered from selection bias due to purposive selection of the households. Selection of squatter settlements from Kathmandu Valley may limit external validity of the findings to other squatter settlements in Nepal. Likewise, the crosssectional nature of the study may not be appropriate to draw an inference on associations. In addition, assessment of drinking habit was based on self-report. It created difficulty in estimating an accuracy of self-reported exposure, because of possibility of conscious under reporting, especially by women. Reference period of 12 months may have posed problems like recall bias in questions related to frequency, contexts, and types of drinking.

\section{Competing interests}

None of the authors have competing interests.

\section{Author contriutions}

PT was involved in conceptualization of the study, analysis of data, and preparation of first draft of manuscript. SRM contributed in analysis of data, interpretation of findings, and reviewing of the manuscript. Final version of manuscript was approved by both the authors.

\section{Acknowledgements}

The authors would like to acknowledge the respondents for their valuable information.

\section{References}

1. World Health Organization. Global status report on alcohol and health-2014: World Health Organization; 2014.

2. World Health Organization. Global status report on alcohol 2004. World Health Organization Geneva; 2004.

3. Wilsnack RW, Vogeltanz ND, Wilsnack SC, Harris TR. Gender differences in alcohol consumption and adverse drinking consequences: cross-cultural patterns. Addiction. 2000;95(2):251-65.

4. Jiafang Z, Jiachun W, Yunxia L, Xiaoxia Q, Ya F. Alcohol abuse in a metropolitan city in China: a study of the prevalence and risk factors. Addiction. 2004;99(9):1103-10.

5. Mohan D, Chopra A, Sethi H. Incidence estimates of substance use disorders in a cohort from Delhi, India. Indian Journal of Medical Research. 2002;115:128. 6. Joffe AH. Alcohol and Social Complexity in Ancient Western Asia 1. Current Anthropology. 1998;39(3):297-322.

7. Holmila M, Raitasalo K. Gender differences in drinking: why do they still exist? Addiction. 2005;100(12):1763-9.

8. Dhital R, Subedi G, Gurung YB, Hamal P. Alcohol and Drug Use in Nepal with reference to children. Kathmandu: Child Workers in Nepal Concerned Centre (CWIN). 2001.

9. Aryal K, Neupane S, Mehata S, Vaidya A, Singh S, Paulin F, et al. Non communicable diseases risk factors: STEPS Survey Nepal 2013. Kathmandu: Nepal Health Research Council. 2014.

10. Oli N, Vaidya A, Thapa G. Behavioural risk factors of noncommunicable diseases among Nepalese Urban poor: A descriptive study from a Slum Area of Kathmandu. Epidemiology Research International. 2013;2013.

11. Jhingan H, Shyangwa P, Sharma A, Prasad K, Khandelwal S. Prevalence of alcohol dependence in a town in Nepal as assessed by the CAGE questionnaire. Addiction. 2003;98(3):339-43.

12. Kumar S, Pokharel B, Nagesh S, Yadav B. Alcohol use among physicians in a medical school in Nepal. 2006.

13. Shrestha NM. Alcohol and drug abuse in Nepal. British journal of addiction. 
1992;87(9):1241-8.

14. Budhathoki N, Shrestha M, Acharya N, Manandhar A. Subantance Use Among Third year Medical Students of Nepal. Journal of Nepal Health Research Council. 2010.

15. Ayah R, Joshi MD, Wanjiru R, Njau EK, Otieno CF, Njeru EK, et al. A population-based survey of prevalence of diabetes and correlates in an urban slum community in Nairobi, Kenya. BMC Public Health. 2013;13(1):371.

16. Singh R, Mukherjee M, Kumar R, Singh R, Pal R. Study of risk factors of coronary heart disease in urban slums of Patna. Nepal Journal of Epidemiology. 2012;2(3):205-12.

17. Waingankar P, Pandit D. A cross sectional study of coronary heart disease in Urban slum population of Mumbai. International Journal of Medical and Clinical Research, ISSN. 2012:0976-5530.

18. Thankappan K, Shah B, Mathur P, Sarma P, Srinivas G, Mini G, et al. Risk factor profile for chronic non-communicable diseases: results of a communitybased study in Kerala, India. 2010.

19. Anand K, Shah B, Yadav K, Singh R, Mathur P, Paul E, et al. Are the urban poor vulnerable to non-communicable diseases? A survey of risk factors for non-communicable diseases in urban slums of Faridabad. National Medical Journal of India. 2007;20(3):115.

20. Wilsnack RW, Wilsnack SC. Gender and alcohol: Individual and social perspectives: Rutgers Center of Alcohol Studies; 1997.

21. Capraro RL. Why college men drink: Alcohol, adventure, and the paradox of masculinity. Journal of American College Health. 2000;48(6):307-15.

22. Patra J, Bakker R, Irving H, Jaddoe VW, Malini S, Rehm J. Dose-response relationship between alcohol consumption before and during pregnancy and the risks of low birthweight, preterm birth and small for gestational age (SGA) - a systematic review and meta-analyses. BJOG: An International Journal of Obstetrics \& Gynaecology. 2011;118(12):1411-21.

23. May PA, Gossage JP. Maternal risk factors for fetal alcohol spectrum disorders: not as simple as it might seem. Alcohol Research \& Health. 2011;34(1):15. 24. O'Leary CM, Nassar N, Kurinczuk JJ, de Klerk N, Geelhoed E, Elliott EJ, et al. Prenatal alcohol exposure and risk of birth defects. Pediatrics. 2010;126(4):e843-e50.

25. Amaro H, Fried LE, Cabral H, Zuckerman B. Violence during pregnancy and substance use. American journal of public health. 1990;80(5):575-9.

26. Wilsnack RW, Wilsnack SC, Klassen AD. Women's drinking and drinking problems: patterns from a 1981 national survey. American journal of public health. 1984;74(11):1231-8.

27. Lumanti Support Group for Shelter (2008). Status of squatter communities along the Bagmati River and its tributaries in the Kathmandu Valley. Highpowered Bagmati Area Sewerage Implementation and Monitoring Committee, Lumanti Support Group for Shelter, Kathmandu.

28. Mahajan B. Methods in Biostatistics. Jaypee Brothers2005.

29. WHO GENACIS. Gender, alcohol, and culture: an international study (genacis) : core questionaire 2001. [cited 2014 July 4]; Available from: http:// www.genacis.org/questionnaires/core.pdf.

30. Benegal V, Nayak M, Murthy P, Chandra P, Gururaj G, Obot I, et al. Women and alcohol use in India. Alcohol, gender and drinking problems: Perspectives from low and middle income countries. 2005:89-123.

31. Ibanga A, Adetula A, Dagona Z, Karick H, Ojiji O, Obot I, et al. The contexts of alcohol consumption in Nigeria. Alcohol, gender and drinking problems: Perspectives from low and middle income countries. 2005:143-66.

32. MUNNÉ MI. Social consequences of alcohol consumption in Argentina. Alcohol, Gender and Drinking Problems. 2005:25.

33. Room R, Jernigan D, Carlini-Marlatt B, Gureje O, Mäkelä K, Marshall $\mathrm{M}$, et al. Alcohol in developing societies: a public health approach: Finnish Foundation for Alcohol Studies; 2002.

34. Wilsnack RW, Wilsnack SC, Obot IS. Why study gender, alcohol and culture. Alcohol, Gender and Drinking Problems. 2005:1.

35. Brienza RS, Stein MD. Alcohol use disorders in primary care. Journal of general internal medicine. 2002;17(5):387-97.

36. Berg MJ, Kremelberg D, Dwivedi P, Verma S, Schensul JJ, Gupta K, et al. The effects of husband's alcohol consumption on married women in three lowincome areas of greater Mumbai. AIDS and Behavior. 2010;14(1):126-35.

37. Oshiro A, Poudyal AK, Poudel KC, Jimba M, Hokama T. Intimate partner violence among general and urban poor populations in Kathmandu, Nepal. Journal of interpersonal violence. 2010.

38. Alwan A. Global status report on noncommunicable diseases 2010: World Health Organization; 2011.

39. Kalichman SC, Simbayi LC, Kaufman M, Cain D, Jooste S. Alcohol use and sexual risks for HIV/AIDS in sub-Saharan Africa: systematic review of empirical findings. Prevention science. 2007;8(2):141-51.

40. Lim SS, Vos T, Flaxman AD, Danaei G, Shibuya K, Adair-Rohani H, et al. A comparative risk assessment of burden of disease and injury attributable to 67 risk factors and risk factor clusters in 21 regions, 1990-2010: a systematic analysis for the Global Burden of Disease Study 2010. The lancet. 2013;380(9859):222460.

41. World Health Organization: Global status report on alcohol and health Geneva: Department of Mental Health and Substance Abuse; 2011.

42. Thapa P, Mishra S, Bista B, Dhungana R, Adhikari N, Soti L, et al. Social Consequences of Alcohol Use among Urban Poor: A Cross-sectional Study in Kathmandu Valley. Journal of Nepal Health Research Council. 2015.

43. World Health Organization. Addressing health of the urban poor in SouthEast Asia Region: challenges and opportunities: World Health Organization; 2011. 